\title{
Hypoxia-induced angiopoietin-like protein 4 as a clinical biomarker and treatment target for human prostate cancer
}

\author{
SHINRO HATA, TAKEO NOMURA, KAZUNORI IWASAKI, RYUTA SATO, \\ MUTSUSHI YAMASAKI, FUMINORI SATO and HIROMITSU MIMATA
}

Department of Urology, Oita University Faculty of Medicine, Yufu, Oita 879-5593, Japan

Received December 26, 2016; Accepted May 18, 2017

DOI: 10.3892/or.2017.5669

\begin{abstract}
Angiopoietin-like protein 4 (ANGPTL4) is a multifunctional protein, playing roles in glucose and lipid metabolism, inflammation, angiogenesis, and tumorigenesis. Recent research suggests that ANGPTL4 is induced by hypoxia and is a useful diagnostic or prognostic marker for various cancers. However, it remains unclear whether ANGPTL4 expression influences prostate cancer. Here we examined the biological and clinical relevance of ANGPTL4 expression in prostate cancer. Firstly we examined ANGPTL4 expression in the prostate cancer cell lines LNCaP and LNCaP/ $\mathrm{CH}$ incubated at $1 \% \mathrm{O}_{2}$ for at least 6 months. We compared cellular proliferation, migration, and ANGPTL4 secretion in a culture medium between these cell lines. In addition, we investigated the effect of various concentrations of recombinant ANGPTL4 protein (rANGPTL4) on cellular proliferation and intracellular signaling pathways. Moreover, we used ANGPTL4 knockdown by RNA interference to investigate the influence of ANGPTL4 expression on these cell lines. Finally, we investigated the correlation between ANGPTL4 expression in prostate cancer specimens and clinicopathological parameters using immunohistochemistry. Our data suggested that the expression of ANGPTL4 in hypoxic conditions was 14.4-fold higher than that in normoxic condition. ANGPTL4 secretion in the culture medium increased 7.0-fold. In addition, rANGPTL4 increased cellular proliferation 1.72-fold via Akt activation. Moreover, ANGPTL4 knockdown decreased cell growth and its secretion by 25.7 and $41.4 \%$, respectively, compared with the control. A multivariate analysis showed that positive ANGPTL4 expression in the resected specimens was an independent prognostic indicator of biochemical recurrence $(\mathrm{P}=0.03$, hazard ratio $=2.02)$. Our results show
\end{abstract}

Correspondence to: Dr Shinro Hata, Department of Urology, Oita University Faculty of Medicine, 1-1 Idaigaoka, Hasama-machi, Yufu, Oita 879-5593, Japan

E-mail: hatashinn@oita-u.ac.jp

Key words: angiopoietin-like protein 4, prostate cancer, prostatectomy, biochemical recurrence, hypoxia that ANGPTL4 is induced by hypoxia and promotes cancer progression via the activated PI3K/Akt pathway. Moreover, ANGPTL4 can be used as a prognostic marker for prostate cancer patients undergoing radical prostatectomy.

\section{Introduction}

Prostate cancer was the second most frequently diagnosed cancer in 2012 (1) and the sixth leading cause of death due to cancer in males worldwide (2). Research into the pathways of prostate cancer growth suggests a novel therapeutic strategy to enhance the survival of this group (3).

Since vessels do not form in tumors, adequate gas exchange does not occur between the tumor cells and surrounding environment. This leads to intratumoral hypoxia, known to promote cancer cell proliferation, invasion, metastasis, and resistance to radiation and chemotherapy $(4,5)$. We previously reported that chronic hypoxia induces androgen-independent growth in the human prostate cancer cell line LNCaP (6). Hypoxic conditions upregulate the expression of some proteins including angiopoietin-like protein 4 (ANGPTL4).

ANGPTL4 is a member of the angiopoietin family and is highly expressed in adipose and gastrointestinal tissues $(7,8)$. Furthermore, ANGPTL4 is also known to influence inflammation, angiogenesis, and tumorigenesis (9-12). Several studies have reported a role of ANGPTL4 in cancer development, and its suppression can impair tumor growth by enhancing apoptosis (13). Considering the fact that hypoxia is a feature of the tumor microenvironment (5), it has been suggested that ANGPTL4 has effects on prostate cancer growth and/or malignant behavior. However, knowledge on the function of ANGPTL4 in human prostate cancer remains limited (14).

Thus in the present study, we examined ANGPTL4 expression in prostate cancer cell lines under hypoxia and investigated the link between ANGPTL4 expression and associated clinicopathological factors.

\section{Materials and methods}

Cell cultures. We purchased a human-androgen-dependent prostate cancer cell line ( $\mathrm{LNCaP}$ ) from the American Type Culture Collection (Rockville, MD, USA). These cells were cultured in RPMI-1640 medium (Sigma-Aldrich, St. Louis, MO, USA) supplemented with $15 \%$ fetal bovine serum (FBS, 
Sigma-Aldrich), $50 \mathrm{IU} / \mathrm{ml}$ penicillin, and $50 \mathrm{mg} / \mathrm{ml}$ streptomycin sulfate (Gibco, Grand Island, NY, USA) at $37^{\circ} \mathrm{C}$ with $5 \% \mathrm{CO}_{2}$ and $95 \%$ room air (normoxia), or $5 \% \mathrm{CO}_{2}, 94 \% \mathrm{~N}_{2}$, and $1 \% \mathrm{O}_{2}$ (hypoxia). LNCaP/CH cells were cultured under hypoxia for at least 6 months.

Proliferation assay. The cells were seeded into 12-well plates at a density of $1 \times 10^{5}$ cells per well. They were then trypsinized, collected, and counted using a hemocytometer 24,48 , and $72 \mathrm{~h}$ after seeding.

Water-soluble tetrazolium salt (WST-1) assay. The cells were seeded into 96-well plates at density of 5,000 cells per well. After culturing overnight, a fresh standard medium containing various concentrations of ANGPTL 4 recombinant proteins (rANGPTL4) (0-1000 pg/ml) was added to each well. After incubation for $48 \mathrm{~h}, 5 \mathrm{mM}$ WST-1 (Dojindo, Kumamoto, Japan) and $3 \mathrm{nM}$ 1-methoxy-5-methylphenazinium methyl sulfate (Dojindo) were added to each well. After incubation for 1-4 h, absorbance was measured at $450 \mathrm{~nm}$ with a microplate reader (Tecan Japan Co., Ltd., Kanagawa, Japan).

Cell migration assay. Cell migration was assessed using a 24-well plate with Corning ${ }^{\circledR}$ FluoroBlok Inserts (Corning Inc., Corning, NY, USA). The cells were seeded at $5 \times 10^{4}$ cells per well in $0.5-\mathrm{ml}$ serum-free medium. The outer chambers were filled with $0.75 \mathrm{ml}$ of media containing $15 \%$ FBS. After 24 or 48 h, migrating cells were labeled using Cellstain ${ }^{\circledR}$ Calcein-AM solution (Dojindo). After incubation for $1 \mathrm{~h}$ under normoxia or hypoxia, the cell fluorescence was detected using a microplate reader.

RNA isolation and quantitative real-time PCR. This method was performed as previously described (6). The primers comprised ANGPTL4 (Hs_ANGPTL4_1_SG QuantiTect Primer Assay, QT00003631) and $\beta$-actin (HS_ACTB_1_SG QuantiTect Primer Assay, QT00095431). The quantification of mRNA expression was normalized using $\beta$-actin.

Quantification of ANGPTL4 protein by ELISA. The cells were cultured in six-well plates at a density of $5 \times 10^{5}$ cells per well for 24,48 , and $72 \mathrm{~h}$. The medium was then collected before adding fresh medium to the cultures. The collected medium was used to quantify ANGPTL4 protein concentration using a commercial ELISA according to the manufacturer's instructions (Human ANGPTL4 ELISA; Raybiotech, Inc., Norcross, GA, USA).

Establishment of ANGPTL4-overexpressing cells. To create stably transfected lines, LNCaP cells were transduced with either FLAG-tagged ANGPTL4-expressing (Addgene) or control vectors (Cell Biolabs, Inc., San Diego, CA, USA) using Lipofectamine 3000 (Invitrogen), according to the manufacturer's protocol. The transfected cell lines were selected in $0.2-\mu \mathrm{g} / \mathrm{ml}$ puromycin dihydrochloride (Santa Cruz Biotechnology, Dallas, TX, USA).

Protein extraction and immunoblot analysis. This method was performed as previously described (6). The primary antibodies included anti-Bcl-2 (cat. no. SASC509, Santa Cruz
Table I. Characteristics of the patients and tumors.

\begin{tabular}{|c|c|}
\hline Characteristics & Value \\
\hline Median age, years (range) & $67.5(49-76)$ \\
\hline Median follow-up, months (range) & $53.5(13-82)$ \\
\hline Median PSA at diagnosis, ng/ml (range) & $9.0(3.7-23.9)$ \\
\hline \multicolumn{2}{|l|}{ PSA } \\
\hline$<10$ & 43 \\
\hline$\geq 10$ & 27 \\
\hline \multicolumn{2}{|l|}{$\mathrm{pT}$} \\
\hline$\leq 2$ & 61 \\
\hline$\geq 3$ & 9 \\
\hline \multicolumn{2}{|l|}{ Gleason score } \\
\hline$\leq 6$ & 20 \\
\hline$\geq 7$ & 47 \\
\hline Unremarkable & 3 \\
\hline \multicolumn{2}{|l|}{ Extraprostatic extension } \\
\hline 0 & 50 \\
\hline 1 & 20 \\
\hline \multicolumn{2}{|l|}{ Resection margin } \\
\hline 0 & 54 \\
\hline 1 & 14 \\
\hline Unremarkable & 2 \\
\hline \multicolumn{2}{|l|}{ Biochemical recurrence } \\
\hline- & 51 \\
\hline+ & 19 \\
\hline
\end{tabular}

PSA, prostate-specific antigen.

Biotechnology), anti-Bad (cat. no. 9292T, Cell Signaling), anti-phospho-Akt (Set473; cat. no. 9271S, Cell Signaling), anti-Akt (cat. no. 9272S, Cell Signaling), anti- $\beta$-tubulin (cat. no. \#MAB3408, EMD Millipore), and anti-ANGPTL4 (cat. no. GTX114198, GeneTex).

ANGPTL4 knockdown by RNA interference. LNCaP cells were transiently transfected with an ANGPTL4 siRNA duplex [siANGPTL4, final concentration: $60 \mathrm{nmol} / 1$ (Qiagen Inc.)] or control siRNA [random scrambled sequence: siScr, final concentration: $60 \mathrm{nmol} / \mathrm{l}$ (Qiagen Inc.)] using Lipofectamine RNAiMAX (Invitrogen Life Technologies) according to the manufacturer's instructions. The siRNA sequences against ANGPTL4 generated by Qiagen were 5'-GGGACAAGAACUGCGCCAATT-3' and 5'-UUGGCGCAGUUCUUGUCCCTG -3'.

Flow cytometry analysis (FACS). This method was performed as previously described (6). The cell cycles were analyzed using a BD FACSVerse ${ }^{\mathrm{TM}}$ flow cytometer and BD FACSuite ${ }^{\mathrm{TM}}$ software (Becton Dickinson, San Jose, CA, USA).

Docetaxel or LY294002 treatment. The cells were seeded into 12 -well plates at a density of $1 \times 10^{5}$ cells per well. After culturing 
A

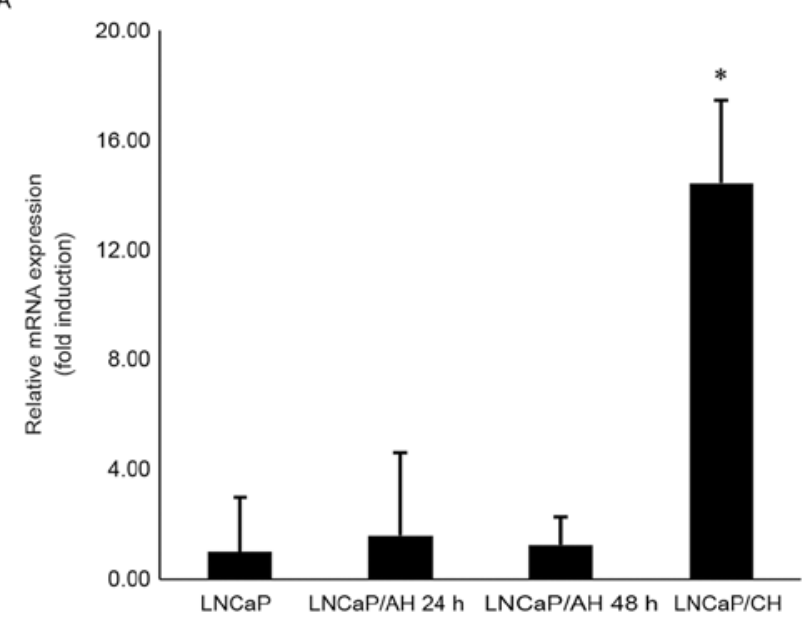

C $(\mathrm{pg} / \mathrm{ml})$

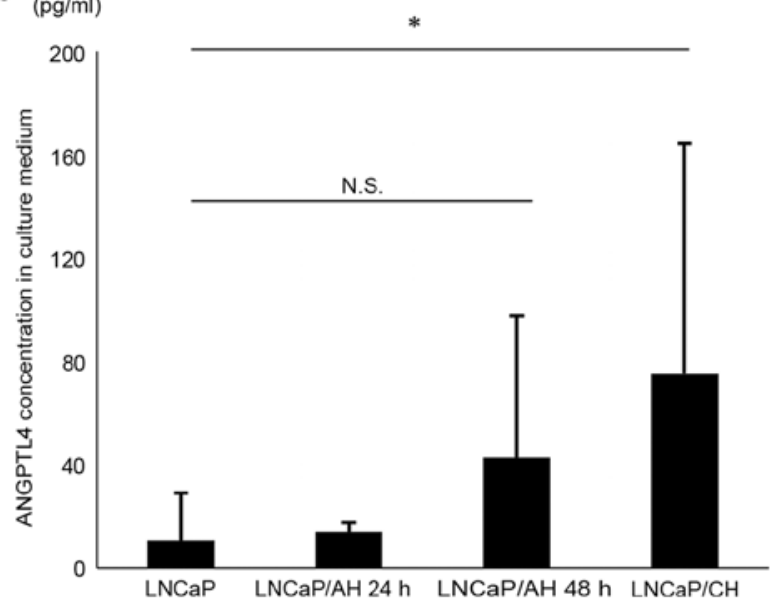

B

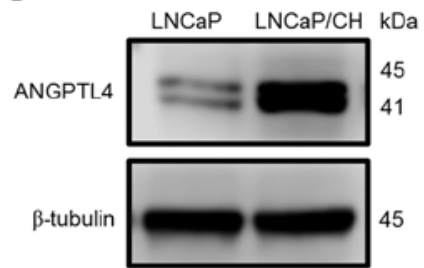

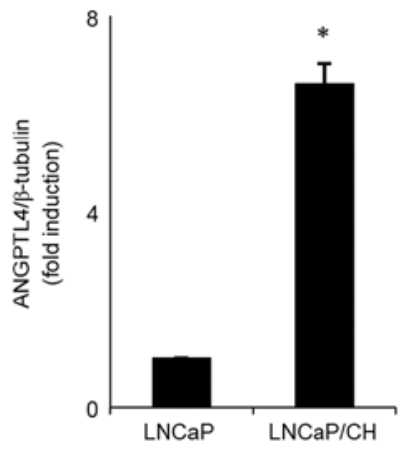

Figure 1. ANGPTL4 expression under chronic hypoxia conditions in LNCaP cells. (A) Relative ANGPTL4 mRNA expression in LNCaP cells. LNCaP/CH cells expressed higher levels of ANGPTL4 than those in LNCaP and LNCaP/AH cells. ${ }^{*}<0.05$ compared with LNCaP and LNCaP/AH cells. (B) The images show the immunoblot results and graphical quantification for ANGPTL4 and $\beta$-tubulin in LNCaP cells. *P<0.05 compared with LNCaP cells. (C) ANGPTL4 secretion levels in LNCaP cells. Higher ANGPTL4 expression is observed in the culture medium of $\mathrm{LNCaP} / \mathrm{CH}$ cells cultured for $72 \mathrm{~h}$ than in LNCaP cells. ${ }^{*} \mathrm{P}<0.05$ compared with LNCaP cells. Values represent mean \pm SD from three independent experiments. ANGPTL4, angiopoietin-like protein 4 ; N.S., not significant.

overnight, a fresh standard medium containing $2 \mathrm{nM}$ docetaxel (Sigma-Aldrich) was added to the plates. The phosphoinositide 3-kinase (PI3K) inhibitor, LY294002, was obtained from Calbiochem (Darmstadt, Germany). Docetaxel is the standard chemotherapy for men with advanced prostate cancer.

Patients. As this study was a retrospective chart review, no patient consent was required. From June 2009 to December 2012 , 92 patients underwent curative surgery involving a radical prostatectomy for localized prostate cancer at Oita University Hospital (Oita, Japan). Patients who received therapy before and/ or immediately after surgery were excluded, and data from the remaining 70 patients were analyzed (Table I). After surgery, the serum prostate specific antigen (PSA) levels were monitored, and PSA recurrence was defined as an elevation in serum PSA levels ( $\geq 0.2 \mathrm{ng} / \mathrm{ml})$ in two consecutive measurements.

Immunohistochemistry. This method was performed as previously described (15). The primary polyclonal goat anti-human ANGPTL4 (cat. no. 18374-1-AP, Proteintech ${ }^{\mathrm{TM}}$ ) antibody diluted to $100 \mathrm{X}$ with PBS containing $1 \%$ bovine serum albumin was applied.

Evaluation of immunostaining. To evaluate ANGPTL4 staining, we counted the proportion of positively stained cells out of 1,000 cells from five randomly selected areas. ANGPTL4 expression was divided according to the percentage of positive tumor cells (negative, $<20 \%$; weak: $20-50 \%$; and strong, $>50 \%$ ). Two authors independently evaluated in a blinded manner to the clinical findings, the level of immunoreactivity using an ECLIPSE E600 research microscope (Nikon Corp., Tokyo, Japan).

Statistical analysis. Statistical analyses were performed using a Student's t-test, Fisher's exact test, Mann-Whitney U-test, or Spearman's rank correlation test. A two-tailed test was used for all analyses. Biochemical recurrence-free survival was estimated using the Kaplan-Meier method and compared the groups using the log-rank test. A multivariate analysis was performed using a Cox's proportional hazards model. The P-values of $<0.05$ were considered to be statistically significant. All statistical analyses were performed using EZR (Saitama Medical Center, Jichi Medical University, Saitama, Japan), a graphical user interface for R (The R Foundation for Statistical Computing, Vienna, Austria) (16).

\section{Results}

ANGPTL4 expression levels during chronic hypoxia. $\mathrm{LNCaP} / \mathrm{CH}$ cells were grown under hypoxic conditions for 


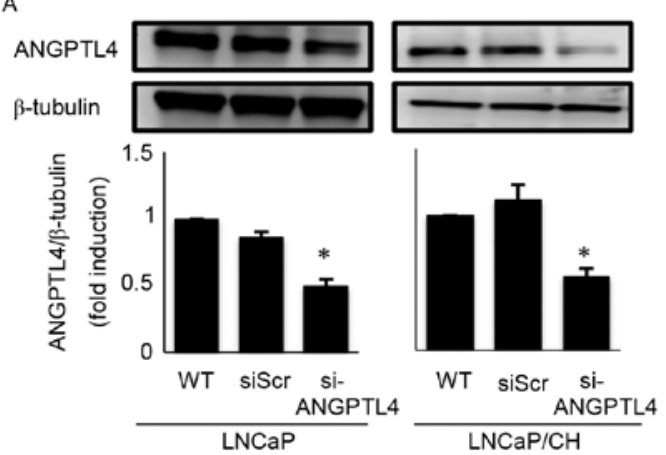

C
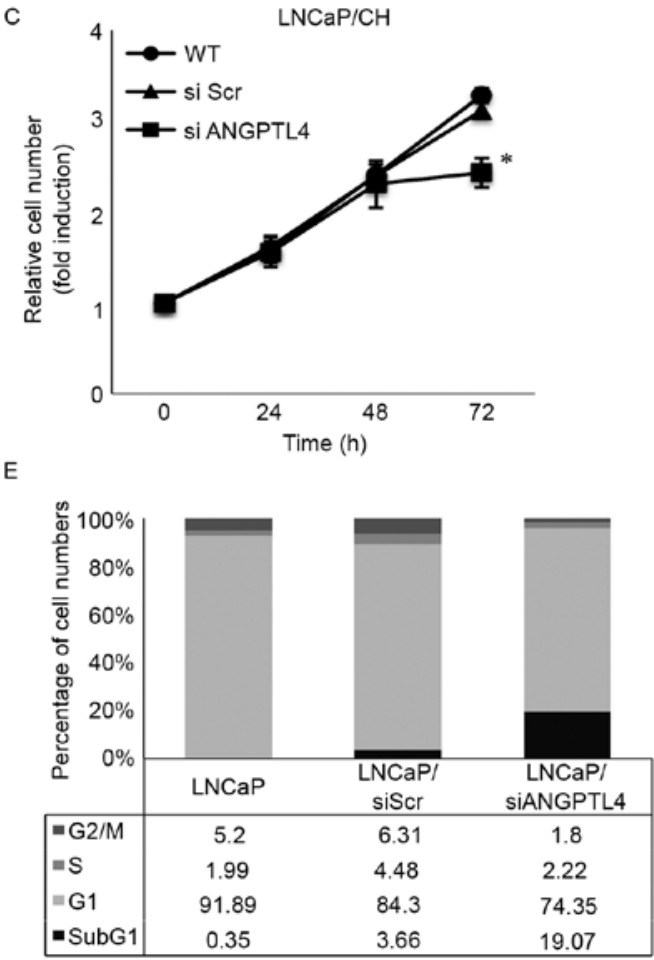

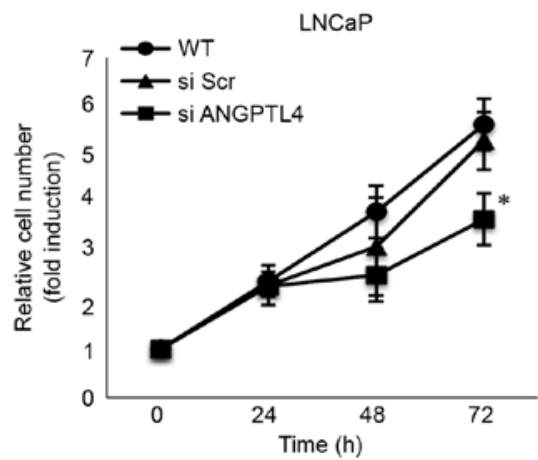

D

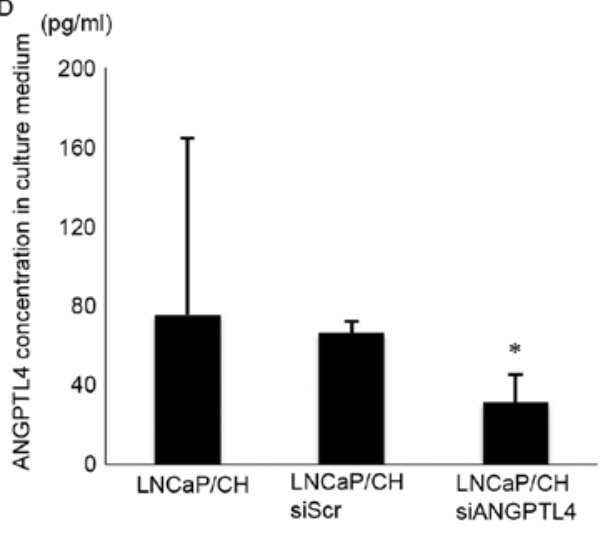

Figure 2. ANGPTL4 knockdown inhibits $\mathrm{LNCaP}$ and $\mathrm{LNCaP} / \mathrm{CH}$ cell growth. (A) The images show the immunoblot results and graphical quantification for ANGPTL4 and $\beta$-tubulin in LNCaP cells and LNCaP/CH cells. In both cell lines, siANGPTL4 effectively downregulated ANGPTL4 expression. "P $<0.05$ compared with WT and siScr-treated cells. (B and C) The effect of siANGPTL4 on the proliferation of LNCaP and LNCaP/CH cells. "P $<0.05$ compared with WT and siScr-treated cells. (D) The effect of siANGPTL4 on ANGPTL4 secretion based on the results of ELISA. "P<0.05 compared with LNCaP/CH cells and LNCaP/CH siScr cells. (E) The effect of siANGPTL4 on the cell cycle. Values represent the mean \pm SD of three independent experiments. ANGPTL4, angiopoietin-like protein 4; WT, wild-type.

over 6 months, and relative ANGPTL4 mRNA expression was 14.4-, 9.0-, and 11.4-fold higher in $\mathrm{LNCaP} / \mathrm{CH}$ cells than in LNCaP, LNCaP/AH $24 \mathrm{~h}$ and LNCaP/AH $48 \mathrm{~h}$ cells, respectively (Fig. 1A). Similarly, ANGPTL4 protein expression was 6.6-fold higher in $\mathrm{LNCaP} / \mathrm{CH}$ cells than in $\mathrm{LNCaP}$ cells (Fig. 1B). Fig. 1B showed a doublet band and the molecular weight of the detected bands was 41 and $45 \mathrm{kDa}$. ANGPTL4 could be cleaved to an active form and the lower band indicates the active form of ANGPTL4.

ANGPTL4 secretion levels during chronic hypoxia. After $72 \mathrm{~h}$, ANGPTL4 secretion was 7.0-fold higher in LNCaP/CH cells than in LNCaP cells (Fig. 1C); however, no statistically significant differences were found after $48 \mathrm{~h}$ (data not shown).

Effect of ANGPTL4 siRNA on LNCAP and LNCAP/CH cell behavior. ANGPTL4 siRNA (siANGPTL4) effectively down- regulated ANGPTL4 expression in both $\mathrm{LNCaP}$ and $\mathrm{LNCaP} /$ $\mathrm{CH}$ cells (Fig. 2A). After incubation for $72 \mathrm{~h}$ with siANGPTL4, cellular proliferation decreased to 82.0 and $74.2 \%$ in $\mathrm{LNCaP}$ and $\mathrm{LNCaP} / \mathrm{CH}$ cells, respectively (Fig. 2B and C). Similarly, siANGPTL4 decreased ANGPTL4 secretion to $41.2 \%$ after $72 \mathrm{~h}$ in LNCaP/CH cells (Fig. 2D). Flow cytometry revealed an increase in hypodiploid DNA (sub-G1 population) cells among the siANGPTL4 transfected cells, concomitant to a significant growth inhibition (Fig. 2E).

ANGPTL4 overexpression accelerates the cellular migration of LNCaP cells. To investigate the precise behavior of ANGPTL4 in prostate cancer, we established a stable cell line overexpressing ANGPTL4 (LNCaP/ANGPTL4). LNCaP/ANGPTL4 increased ANGPTL4 expression by 5.1-fold compared to the control (Fig. 3A). Furthermore, cellular migration was 2.0-fold and 3.0-fold higher in LNCaP/ANGPTL4 cells than in LNCaP 
A

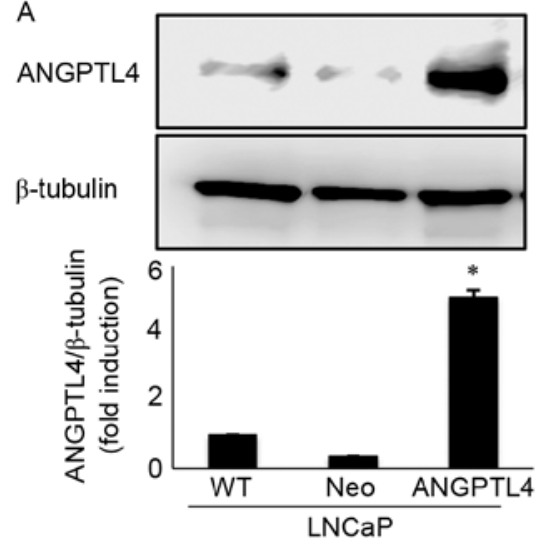

C

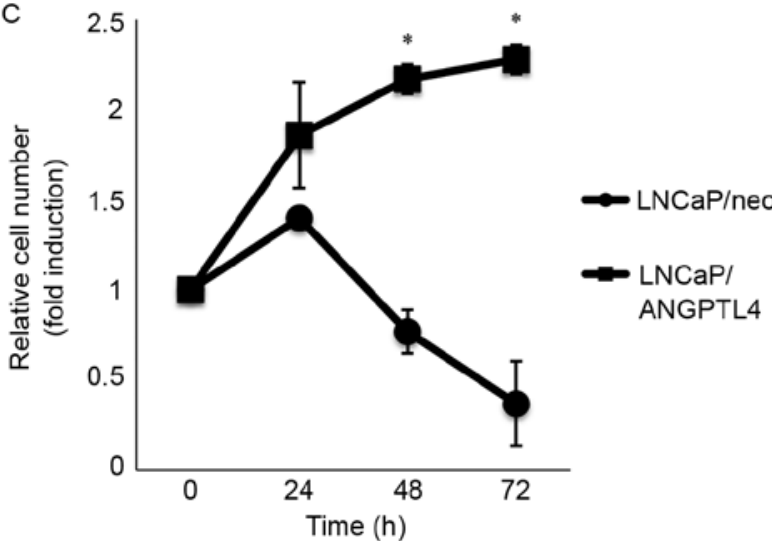

B

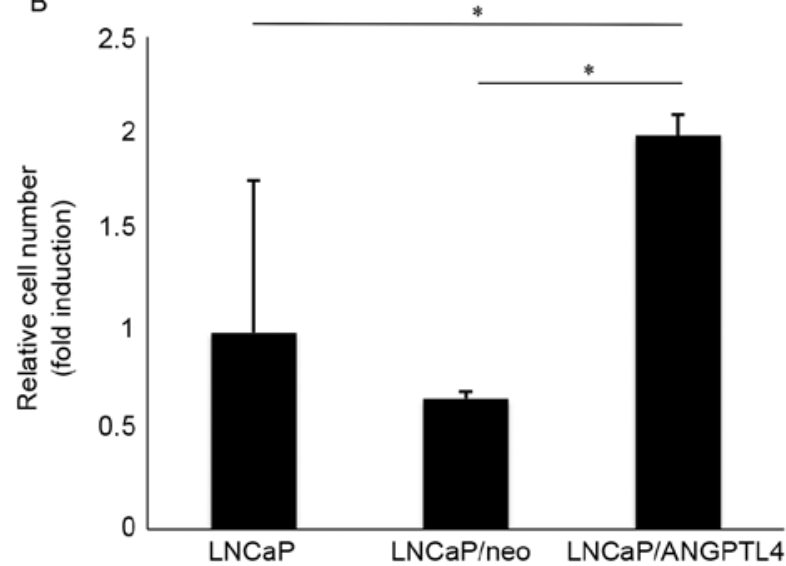

D

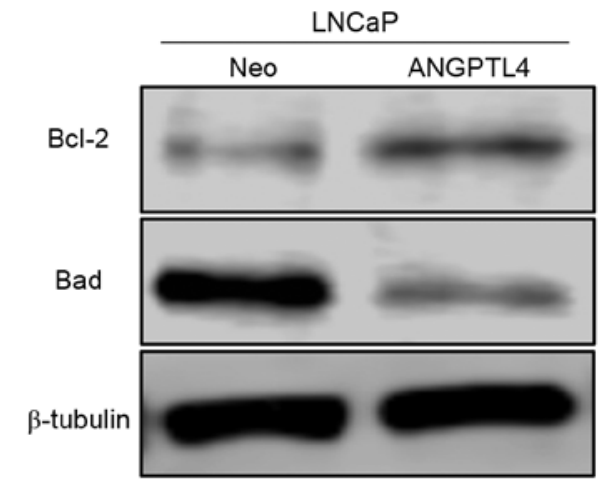

Figure 3. ANGPTL4 overexpression affects tumorigenicity and chemoresistance by inhibiting apoptosis. (A) The images show the immunoblot results and graphical quantification for ANGPTL4 and $\beta$-tubulin in LNCaP cells. LNCaP/ANGPTL4 upregulates ANGPTL4 expression. "P<0.05 compared with LNCaP cells and LNCaP/neo cells. (B) Migration assay of LNCaP, LNCaP/neo, and LNCaP/ANGPTL4 cells. The image shows cell migration after $24 \mathrm{~h}$. "P $<0.05$ compared with $\mathrm{LNCaP}$ cells and $\mathrm{LNCaP} /$ neo cells. (C) The relative proportion of proliferative $\mathrm{LNCaP} /$ neo and LNCaP/ANGPTL4 cells after treatment with docetaxel. ${ }^{~} \mathrm{P}<0.05$ compared with $\mathrm{LNCaP} /$ neo cells. (D) The image shows the immunoblot results for Bcl-2, Bad, and $\beta$-tubulin for $\mathrm{LNCaP} / \mathrm{neo}$ and LNCaP/ANGPTL4 cells. In LNCaP/ANGPTL4 cells, Bcl-2 expression was upregulated, whereas Bad expression was downregulated following treatment with docetaxel. Values represent mean \pm SD from three independent experiments. ANGPTL4, angiopoietin-like protein 4; Bcl-2, B-cell lymphoma 2; Bad, Bcl-2-associated death promoter.

and LNCaP/neo-transfected cells with a control vector, respectively (Fig. 3B). In contrast, cellular proliferation did not differ significantly among the cell lines (data not shown).

ANGPTL4 activates the PI3K/Akt pathway, and LY294002 inhibits the cellular proliferation of LNCaP cells. To assess the potential role of ANGPTL4, we added different concentrations of rANGPTL4 to LNCaP cells (Fig. 4A). Treatment with $100 \mathrm{pg} / \mathrm{ml}$ of rANGPTL4 for $72 \mathrm{~h}$ increased cellular proliferation 1.72-fold (Fig. 4B). The immunoblot assay also revealed that Akt phosphorylation at Ser 473, a site required for Akt activation, was upregulated both time- and dose-dependently (Fig. 4C and D). However, cellular proliferation was suppressed in the cells subsequently treated with LY294002 (a PI3K inhibitor) $6 \mathrm{~h}$ later (Fig. 4E). Akt phosphorylation peaked at 5-15 min (Fig. 4C), but the effect on cellular proliferation was observed 48-72 h later (Fig. 4B). It is considered that with the activation of Akt, various signaling molecules are activated resulting in cell survival.

ANGPTL4 overexpression and drug resistance. Cellular proliferation was successfully repressed in $\mathrm{LNCaP} / \mathrm{neo}$ cells following $2 \mathrm{nM}$ docetaxel treatment; however, $\mathrm{LNCaP} /$ ANGPTL4 cells maintained their proliferative capabilities even after treatment (Fig. 3C). The immunoblot assay revealed that anti-apoptotic B-cell lymphoma 2 (Bcl-2) expression was upregulated and that of Bcl-2-associated death promoter (Bad) was downregulated in LNCaP/ANGPTL4 cells compared with that in the controls (Fig. 3D). Thus, these results indicate that ANGPTL4 expression is linked to docetaxel resistance by inhibiting apoptosis.

Patient characteristics. Table I presents the clinicopathological characteristics of the patients included in this study. The median age of the patients was 67.5 (range: 49-76) years. The median PSA at diagnosis was 9.0 (range: $3.7-23.9$ ) $\mathrm{ng} / \mathrm{ml}$. The pathological $\mathrm{T}(\mathrm{pT})$ stage was $\leq \mathrm{pT} 2$ in 61 cases $(87.1 \%)$ and $\geq \mathrm{pT} 3$ in nine cases (12.9\%). The Gleason score (GS) was $\leq 6$ in 20 cases (28.6\%) and $\geq 7$ in 47 cases $(67.1 \%)$. The remaining three cases were unremarkable. During a median follow-up of 53.5 (range: 13-82) months, 19 patients (27.1\%) had PSA recurrence.

Immunohistochemistry for ANGPTL4. We evaluated ANGPTL4 expression by immunohistochemistry in 70 prostate 

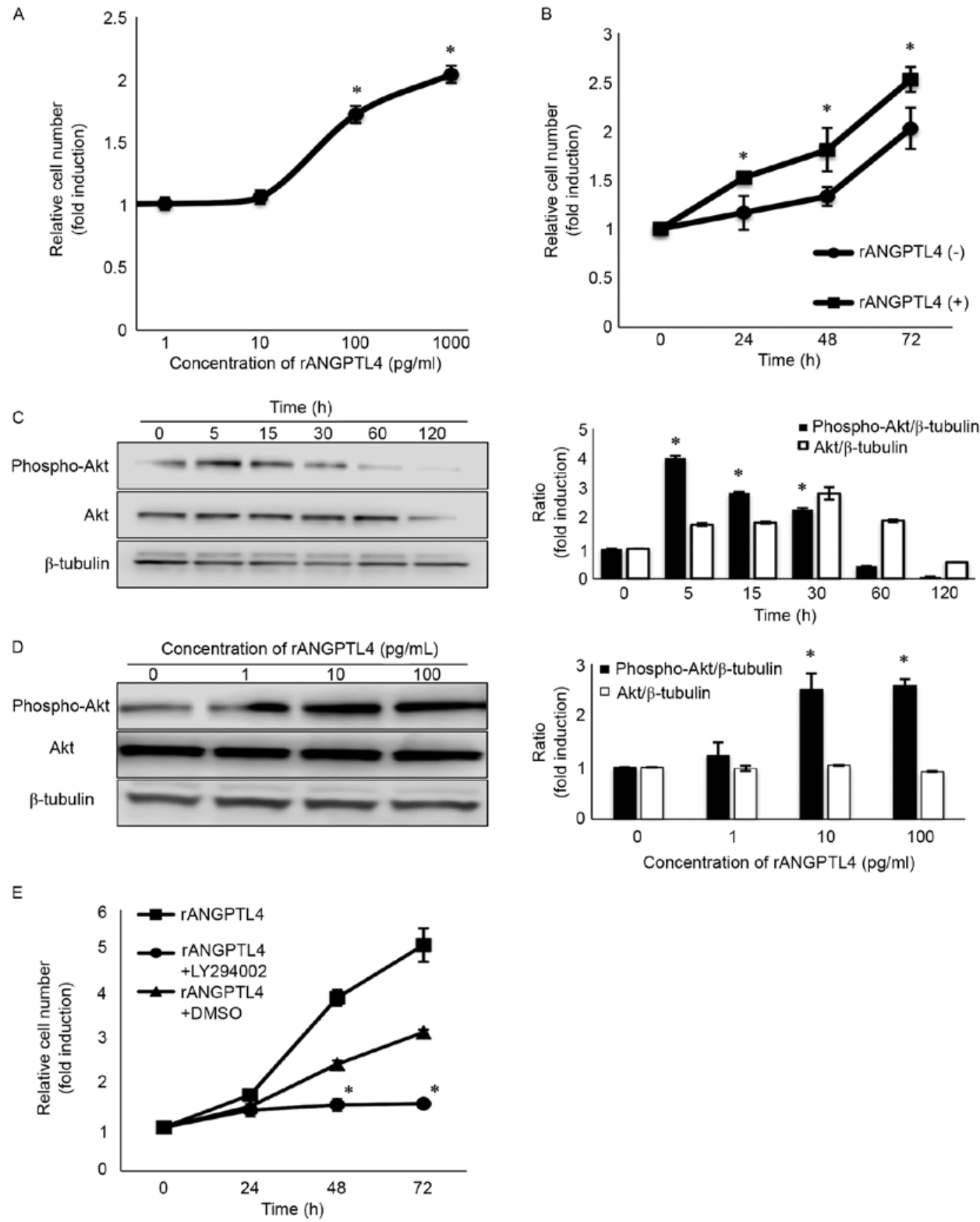

Figure 4. ANGPTL4 induces cellular proliferation through Akt activation. (A) WST-1 assay of LNCaP cells after treatment with rANGPTL4. "P $<0.05$ compared with the control. (B) The relative proportion of proliferative LNCaP cells after treatment with or without rANGPTL4. "P<0.05 compared with LNCaP cells without rANGPTL4. (C and D) The images show the immunoblot results and graphical quantification for a representative image of phospho-Akt, Akt and $\beta$-tubulin after the treatment of LNCaP cells with rANGPTL4. ${ }^{*} \mathrm{P}<0.05$ compared with the control. (E) The relative proportion of proliferative LNCaP cells after treatment with rANGPTL4 with or without LY294002. " $\mathrm{P}<0.05$ compared with the control. Values represent the mean \pm SD from three independent experiments. ANGPTL4, angiopoietin-like protein 4; rANGPTL4, recombinant ANGPTL4 protein.

tissue samples. Normal prostate gland tissue did not express ANGPTL4. In contrast, we observed negative staining in 12 of the 70 prostate cancer samples $(17.1 \%)$ and positive staining in 58 samples $(82.9 \%)$. ANGPTL4 expression was particularly strong in 11 samples. Fig. 5A shows the representative images.

Association between ANGPTL4 expression and clinicopathological parameters. Table II lists the correlation between ANGPTL4 expression levels and different clinicopathological factors that were analyzed. ANGPTL4 expression levels were significantly correlated with PSA recurrence $(\mathrm{P}=0.02)$. However, PSA at diagnosis, GS, pT stage, and the resection margin were not correlated with ANGPTL4 expression. An extraprostatic extension tended to be higher in cells with positive ANGPTL4 expression than in cells with negative ANGPTL4 expression; however, this difference was not significant $(\mathrm{P}=0.091)$.

ANGPTL4 expression levels and patient outcomes. We performed univariate and multivariate analyses to determine 
A
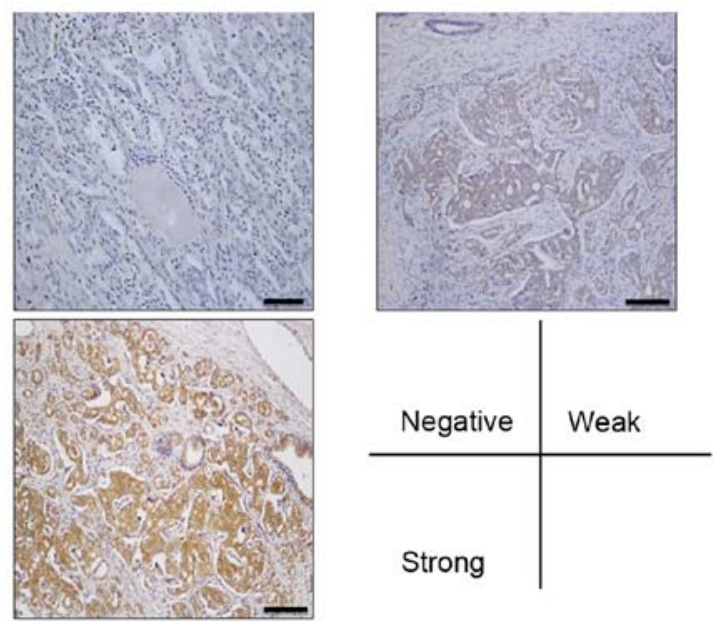

B

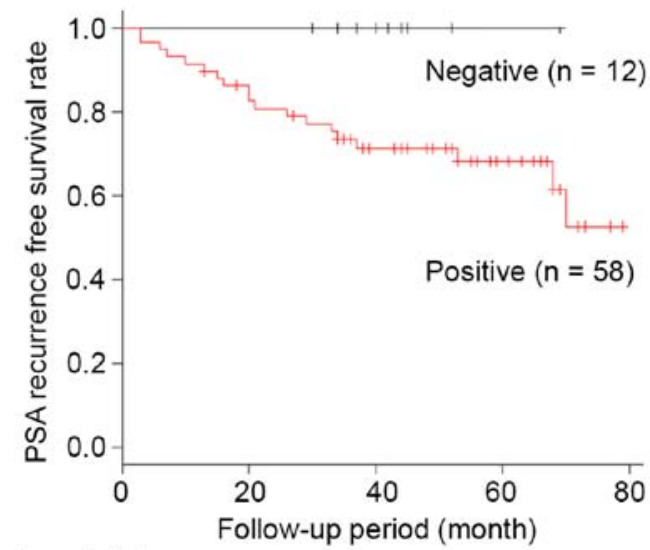

Number at risk
Negative 12

Positive 58

\begin{abstract}
12
\end{abstract}
48

2

0

Figure 5. Immunohistochemical analysis of ANGPTL4 expression in prostate cancer tissues and the Kaplan-Meier curve of biochemical recurrence-free survival based on ANGPTL4 expression. (A) ANGPTL4 expression level was divided into three categories according to the percentage of positive tumor cells (negative, $<20 \%$; weak, 20-50\%; and strong, $>50 \%$ ). (B) Biochemical recurrence-free survival rate estimated using the Kaplan-Meier method and compared using the log-rank test. Probability values of $\mathrm{P}<0.05$ were considered to be statistically significant. ANGPTL4, angiopoietin-like protein 4; PSA, prostate-specific antigen.

the factors that could be used as indicators of PSA recurrence after surgery (Table III). A univariate analysis revealed that both $\mathrm{pT}$ stage $(\mathrm{P}=0.007)$ and positive ANGPTL4 expression of the tumor $(\mathrm{P}=0.04)$ were indicators of PSA recurrence. The multivariate analysis using the Cox's proportional hazards model revealed that positive ANGPTL4 expression was an independent prognostic indicator of PSA recurrence $(\mathrm{P}=0.03$, hazard ratio $=2.02)$. The 3-year PSA recurrencefree survival rate for patients with positive ANGPTL4 expression was significantly lower than that for other patients $(\mathrm{P}=0.01)$ (Fig. 5B).

\section{Discussion}

Herein, we examined ANGPTL4 expression in prostate cancer cell lines under hypoxic conditions. In addition, we assessed the behavior of ANGPTL4 in prostate cancer cell lines. We also assessed whether ANGPTL4 rendered
Table II. Correlation between ANGPTL4 expression and clinicopathological factors.

\begin{tabular}{lcccc}
\hline & \multicolumn{2}{c}{ ANGPTL4 } & & \\
\cline { 2 - 3 } Factors & $\begin{array}{c}\text { Negative } \\
(\mathrm{n}=12)\end{array}$ & $\begin{array}{c}\text { Positive } \\
(\mathrm{n}=58)\end{array}$ & $\mathrm{R}$ & P-value \\
\hline Age, years & & & -0.049 & 0.687 \\
$<65$ & 5 & 21 & & \\
$\geq 65$ & 7 & 37 & & \\
PSA at diagnosis, ng/ml & & & 0.049 & 0.687 \\
$<10$ & 8 & 35 & & \\
$\geq 10$ & 4 & 23 & & \\
Gleason score & & & -0.182 & 0.141 \\
$\leq 6$ & 1 & 17 & & \\
$\geq 7$ & 9 & 40 & & \\
Unremarkable & 2 & 1 & & \\
pT stage & & & 0.166 & 0.172 \\
$\leq 2$ & 12 & 49 & & \\
$\geq 3$ & 0 & 9 & & \\
Extraprostatic extension & & & 0.204 & 0.091 \\
0 & 11 & 39 & & \\
1 & 1 & 19 & & \\
Resection margin & & & -0.051 & 0.683 \\
0 & 9 & 47 & & \\
1 & 3 & 11 & & \\
Biochemical recurrence & & & 0.28 & $\mathbf{0 . 0 2}$ \\
- & 12 & 39 & & \\
+ & 0 & 19 & & \\
\hline
\end{tabular}

ANGPTL4, angiopoietin-like protein 4; PSA, prostate-specific antigen. Bold text indicates statistical significance.

prostate cancer cells resistant to chemotherapy. Finally, we investigated how ANGPTL4 expression correlated with clinicopathological factors.

Our results showed that chronic hypoxia induces ANGPTL4 expression and promotes cancer progression via the activated PI3K/Akt pathway. Previously, we reported that $\mathrm{LNCaP}$ cells conditioned under chronic hypoxia grew in an androgen-independent manner, presented accelerated G1 to S phases of the cell cycle, and accelerated cell migration and invasion. In addition, PI3K/Akt, JAK/STAT, and hypoxiainducible factor 1 (HIF-1) pathways were activated in LNCaP cells conditioned under chronic hypoxia (6). In similar studies, Kim et al reported a synergistic effect of prostaglandin $\mathrm{E}_{2}$ $\left(\mathrm{PGE}_{2}\right)$ and hypoxia on enhancing ANGPTL4 expression, and that increased ANGPTL4 expression promoted colorectal cancer growth. Moreover, these authors showed that hypoxia induced a $\mathrm{PGE}_{2}$ receptor, the E prostanoid receptor (EP) 1. Activation of EP1 enhanced ANGPTL4 expression, whereas blockage of EP1 by an antagonist inhibited $\mathrm{PGE}_{2}$ induction of ANGPTL4 under hypoxic conditions (17). Thus, our 
Table III. Univariate and multivariate analysis of PSA recurrencefree rates.

\begin{tabular}{|c|c|c|c|}
\hline \multirow[b]{3}{*}{ Factors } & \multicolumn{3}{|c|}{ Recurrence-free rates } \\
\hline & \multirow{2}{*}{$\frac{\text { Univariate }}{\text { P-value }}$} & \multicolumn{2}{|c|}{ Multivariate } \\
\hline & & $\mathrm{HR}(95 \% \mathrm{CI})$ & P-value \\
\hline $\begin{array}{l}\text { Age } \\
<65 \\
\geq 65\end{array}$ & 0.244 & & \\
\hline $\begin{array}{l}\text { PSA at diagnosis } \\
<10 \\
\geq 10\end{array}$ & 0.497 & & \\
\hline $\begin{array}{l}\text { Gleason score } \\
\leq 6 \\
\geq 7\end{array}$ & 0.119 & & \\
\hline $\begin{array}{l}\mathrm{pT} \\
\leq 2 \\
\geq 3\end{array}$ & 0.007 & $3.95(0.76-20.54)$ & 0.1 \\
\hline $\begin{array}{c}\text { Extra prostatic extension } \\
0 \\
1\end{array}$ & 0.181 & & \\
\hline $\begin{array}{l}\text { Resection margin } \\
0 \\
1\end{array}$ & 0.393 & & \\
\hline $\begin{array}{l}\text { ANGPTL4 } \\
-\end{array}$ & 0.04 & & 0.032 \\
\hline+ & & $2.02(1.06-3.86)$ & \\
\hline
\end{tabular}

HR, hazard ratio; CI, confidence interval; ANGPTL4, angiopoietinlike protein 4; PSA, prostate-specific antigen. Bold indicates statistical significance.

combined results suggest that ANGPTL4 plays an important role in cancer progression under hypoxia.

In addition, our results showed that ANGPTL4 overexpression plays a role in drug resistance via the anti-apoptotic effect. A correlation between hypoxia and chemoresistance has been previously reported in a number of tumor cell types (18). To this end, many reports have suggested that HIF-1-induced genes mediated chemoresistance either directly or indirectly (19). Furthermore, some studies have reported that hypoxia induced the expression of ANGPTL4, and that the upregulation of ANGPTL4 is induced by the transcription factor HIF-1 $(20,21)$. Our results complement previous research, and they show that ANGPTL4 directly mediates chemoresistance by inhibiting apoptosis in prostate cancer.

Negative ANGPTL4 expression was significantly related to longer PSA recurrence-free survival rates. To the best of our knowledge, these results are the first that suggest ANGPTL4 as a novel marker for PSA recurrence after radical prostatectomy. Generally, curative surgery or radiation therapy is used to treat localized prostate cancer. However, $25-35 \%$ of the patients develop evidence of biochemical recurrence after radical prostatectomy for clinically localized prostate cancer $(22,23)$. Given the increased incidence of prostate cancer and the continued need for improved diagnostic markers to detect prostate cancer recurrence, research has focused on looking for specific blood and tumor markers. Our data indicate that ANGPTL4 can be used as a new prognostic marker. In the same analysis, other standard factors such as pT stage, PSA at diagnosis, and GS were not significantly associated with PSA recurrence. However, this lack of association might be related to the small sample size, and the fact that the patients who immediately received neo-adjuvant or adjuvant therapy after surgery, based on pathological findings, were excluded from the study potentially limited the scope of our results.

The role of ANGPTL4 in cancer remains controversial. For example, $\mathrm{Ng}$ et al reported that ANGPTL4 could represent a potential therapeutic agent to suppress hepatocellular carcinoma growth, angiogenesis, and metastasis (24). On the other hand, a series of studies have reported the role of ANGPTL4 in promoting cancer progression $(12,25,26)$. Previously, ANGPTL4 has been shown to promote tumor cells for lung metastasis in breast cancer patients, and to trigger the disruption of vascular endothelial cell-cell junctions (27). The origin of the discrepancy among these results is also unclear. The inferred cause is that ANGPTL4 is degraded in the human body. ANGPTL4 contains an N-terminal coiled domain (nANGPTL4) and a C-terminal fibrinogen-like domain (cANGPTL4); however, little is known about the relative expression of different ANGPTL4 fragments in each tissue. To this end, a recent study shows that cANGPTL4, but not nANGPTL4, is highly expressed in major epithelial tumors such as adenocarcinoma. The study examined ANGPTL4 expression using immunofluorescence with a monoclonal antibody against cANGPTL4 (mAb11F6C4) (13). Thus, post-translational modifications of ANGPTL4 such as $\mathrm{N}$-glycosylation might be behind different roles played by this protein in various cancers $(28,29)$. The number of uncertainties regarding the role and biological mechanism of ANGPTL4 in human prostate cancer highlights the need of further studies in this front.

In conclusion, we show that hypoxia-induced ANGPTL4 promotes prostate cancer progression via the activated PI3K/Akt pathway. In addition, ANGPTL4 expression in surgically resected prostate cancer specimens can represent a novel prognostic marker. Finally, ANGPTL4 could also be suggested as a potential novel therapeutic target in prostate cancer.

\section{Acknowledgements}

We thank Ms. N. Hamamatsu and Ms. S. Kato for technical assistance in this work. The authors would like to thank Enago (www.enago.jp) for the English language review.

\section{References}

1. Stewart BW and Wild CP (eds): World Cancer Report 2014. International Agency for Research on Cancer, World Health Organization, 2014.

2. Jemal A, Bray F, Center MM, Ferlay J, Ward E and Forman D: Global cancer statistics. CA Cancer J Clin 61: 69-90, 2011.

3. Scardino PT, Marston Linehan W,Zelefsky MJ and Vogelzang NJ: Comprehensive Textbook of Genitourinary Oncology. 4th edition. Lippincott Williams and Wilkins, Philadelphia, PA, 2011. 
4. Höckel M and Vaupel P: Tumor hypoxia: Definitions and current clinical, biologic, and molecular aspects. J Natl Cancer Inst 93: 266-276, 2001.

5. Harris AL: Hypoxia - a key regulatory factor in tumour growth. Nat Rev Cancer 2: 38-47, 2002.

6. Yamasaki M, Nomura T, Sato F and Mimata H: Chronic hypoxia induces androgen-independent and invasive behavior in LNCaP human prostate cancer cells. Urol Oncol 31: 1124-1131, 2013.

7. Tan MJ, Teo Z, Sng MK, Zhu P and Tan NS: Emerging roles of angiopoietin-like 4 in human cancer. Mol Cancer Res 10: 677-688, 2012.

8. Zhu P, Goh YY, Chin HF, Kersten S and Tan NS: Angiopoietin-like 4: A decade of research. Biosci Rep 32: 211-219, 2012.

9. Kersten S, Lichtenstein L, Steenbergen E, Mudde K, Hendriks HF Hesselink MK, Schrauwen P and Müller M: Caloric restriction and exercise increase plasma ANGPTL4 levels in humans via elevated free fatty acids. Arterioscler Thromb Vasc Biol 29: 969-974, 2009

10. Mandard S, Zandbergen F, van Straten E, Wahli W, Kuipers F, Müller M and Kersten S: The fasting-induced adipose factor/ angiopoietin-like protein 4 is physically associated with lipoproteins and governs plasma lipid levels and adiposity. J Biol Chem 281: 934-944, 2006.

11. Lu B, Moser A, Shigenaga JK, Grunfeld C and Feingold KR: The acute phase response stimulates the expression of angiopoietin like protein 4. Biochem Biophys Res Commun 391: 1737-1741, 2010.

12. Kubo H, Kitajima Y, Kai K, Nakamura J, Miyake S, Yanagihara K, Morito K, Tanaka T, Shida M and Noshiro H: Regulation and clinical significance of the hypoxia-induced expression of ANGPTL4 in gastric cancer. Oncol Lett 11: 1026-1034, 2016.

13. Zhu P, Tan MJ, Huang RL, Tan CK, Chong HC, Pal M, Lam CR, Boukamp P, Pan JY, Tan SH, et al: Angiopoietin-like 4 protein elevates the prosurvival intracellular $\mathrm{O}_{2}(-): \mathrm{H}_{2} \mathrm{O}_{2}$ ratio and confers anoikis resistance to tumors. Cancer Cell 19: 401-415, 2011.

14. Ifon ET, Pang AL, Johnson W, Cashman K, Zimmerman S, Muralidhar S, Chan WY, Casey J and Rosenthal LJ: U94 alters FN1 and ANGPTL4 gene expression and inhibits tumorigenesis of prostate cancer cell line PC3. Cancer Cell Int 5: 19-31, 2005.

15. Sato R, Yamasaki M, Hirai K, Matsubara T, Nomura T, Sato F and Mimata H: Angiopoietin-like protein 2 induces androgenindependent and malignant behavior in human prostate cancer cells. Oncol Rep 33: 58-66, 2015.

16. Kanda Y: Investigation of the freely available easy-to-use software 'EZR' for medical statistics. Bone Marrow Transplant 48: 452-458, 2013.

17. Kim SH, Park YY, Kim SW, Lee JS, Wang D and DuBois RN ANGPTL4 induction by prostaglandin $\mathrm{E}_{2}$ under hypoxic conditions promotes colorectal cancer progression. Cancer Res 71: 7010-7020, 2011.
18. Rohwer $\mathrm{N}$ and Cramer T: Hypoxia-mediated drug resistance: Novel insights on the functional interaction of HIFs and cell death pathways. Drug Resist Updat 14: 191-201, 2011.

19. Karakashev SV and Reginato MJ: Progress toward overcoming hypoxia-induced resistance to solid tumor therapy. Cancer Manag Res 7: 253-264, 2015

20. Zhang H, Wong CC, WeiH, Gilkes DM, Korangath $P$, Chaturvedi $P$, Schito L, Chen J, Krishnamachary B, Winnard PT Jr, et al: HIF-1-dependent expression of angiopoietin-like 4 and L1CAM mediates vascular metastasis of hypoxic breast cancer cells to the lungs. Oncogene 31: 1757-1770, 2012.

21. Li H, Ge C, Zhao F, Yan M, Hu C, Jia D, Tian H, Zhu M, Chen T, Jiang $\mathrm{G}$, et al: Hypoxia-inducible factor 1 alpha-activated angiopoietin-like protein 4 contributes to tumor metastasis via vascular cell adhesion molecule-1/integrin $\beta 1$ signaling in human hepatocellular carcinoma. Hepatology 54: 910-919, 2011.

22. Hull GW, Rabbani F, Abbas F, Wheeler TM, Kattan MW and Scardino PT: Cancer control with radical prostatectomy alone in 1,000 consecutive patients. J Urol 167: 528-534, 2002.

23. Roehl KA, Han M, Ramos CG, Antenor JA and Catalona WJ: Cancer progression and survival rates following anatomical radical retropubic prostatectomy in 3,478 consecutive patients: Long-term results. J Urol 172: 910-914, 2004

24. Ng KT, Xu A, Cheng Q, Guo DY, Lim ZX, Sun CK, Fung JH, Poon RT, Fan ST, Lo CM, et al: Clinical relevance and therapeutic potential of angiopoietin-like protein 4 in hepatocellular carcinoma. Mol Cancer 13: 196-212, 2014.

25. Nakayama T, Hirakawa H, Shibata K, Nazneen A, Abe K, Nagayasu T and Taguchi T: Expression of angiopoietin-like 4 (ANGPTL4) in human colorectal cancer: ANGPTL4 promotes venous invasion and distant metastasis. Oncol Rep 25: 929-935, 2011.

26. Shibata K, Nakayama T, Hirakawa H, Hidaka S and Nagayasu T: Clinicopathological significance of angiopoietin-like protein 4 expression in oesophageal squamous cell carcinoma. J Clin Pathol 63: 1054-1058, 2010.

27. Padua D, Zhang XH, Wang Q, Nadal C, Gerald WL, Gomis RR and Massagué J: TGFbeta primes breast tumors for lung metastasis seeding through angiopoietin-like 4. Cell 133: 66-77, 2008.

28. Kim I, Kim HG, Kim H, Kim HH, Park SK, Uhm CS, Lee ZH and Koh GY: Hepatic expression, synthesis and secretion of a novel fibrinogen/angiopoietin-related protein that prevents endothelial-cell apoptosis. Biochem J 346: 603-610, 2000.

29. Yang YH, Wang Y, Lam KS, Yau MH, Cheng KK, Zhang J, Zhu W, Wu D and Xu A: Suppression of the Raf/MEK/ERK signaling cascade and inhibition of angiogenesis by the carboxyl terminus of angiopoietin-like protein 4 . Arterioscler Thromb Vasc Biol 28: 835-840, 2008. 